\title{
La régionalisation de l'immigration : un maillon essentiel d'occupation et de développement des territoires
}

\author{
R. Mathieu Vigneault \\ Directeur général Place aux jeunes en région
}

\section{INTRODUCTION}

\section{La mobilité internationale et interrégionale des personnes est devenue un indicateur de santé socioéconomique et un important facteur de développement.}

Le renversement de la pyramide démographique est l'un des enjeux - sinon le plus grand - auxquels fera face l'ensemble des nations et des États occidentaux. Le Québec ne fait pas exception : il se dirige même plus rapidement que les autres vers un inquiétant déséquilibre sociodémographique, les régions à caractère rural en tête. C'est pourquoi, selon plusieurs experts, la mobilité internationale et interrégionale des personnes est devenue un indicateur de santé socioéconomique et un important facteur de développement. Il n'est donc pas étonnant de constater que le développement démographique et la question de l'attractivité des territoires soient devenus une priorité pour la majorité des régions du Québec. C'est dans cet esprit que l'Assemblée nationale a adopté, le 5 avril 2012, une loi-cadre pour assurer l'occupation et la vitalité des territoires, dont les deux principaux objectifs sont d'agir pour mieux habiter nos territoires et d'agir pour vivre de nos territoires.

\section{L'ENJEU DÉMOGRAPHIQUE EN RÉGION}

Le phénomène dit de «l'exode rural », perçu comme une crise au cours des années 1990, a considérablement changé depuis. Bien entendu, l'amélioration du bilan migratoire des municipalités à caractère rural demeure un défi. Toutefois, c'est avec enthousiasme que nous constatons un certain renversement, du moins dans les perceptions, les discours et les actions. Aujourd'hui, de plus en plus de gens affirment leur fierté d'être natifs de tel ou tel coin de pays, ou leur
Encore aujourd'hui, le retour dans leur milieu d'origine constitue un scénario de choix pour plusieurs jeunes ruraux. Cependant, il demeure que, pour des raisons sociales, économiques, financières, etc., la pérennité de ces communautés ne peut s'appuyer essentiellement sur cette avenue.

L'avenir de la ruralité québécoise (30\% du PIB du Québec) appelle à un effort de créativité, notamment dans l'aménagement du territoire et dans l'organisation du « vivre ensemble », mais aussi - et surtout - dans l'ouverture à une transformation du portrait de la grande famille rurale par l'accueil et par l'intégration d'une immigration volontaire. Cela dit, il apparaît que cette manne sociodémographique amène son lot de défis complexes et de solutions contradictoires.

La question de la place de l'immigration dans la société québécoise est donc appelée à devenir ou plutôt à redevenir un des thèmes d'actualité, surtout que le gouvernement du Québec ambitionne d'adopter une nouvelle politique de l'immigration, de la diversité et de l'inclusion. La question du déploiement de ce précieux capital humain en région risque donc d'interpeler plusieurs décideurs et développeurs régionaux

intention de s'y établir ou de retourner s'y établir un jour.

Il s'agit là d'une situation bien différente de celle des années 1990, alors qu'était colportée une image plutôt morose et défaitiste des régions, parfois par les ruraux eux-mêmes. Depuis 2006, Place aux jeunes en région (PAJR), une organisation dont la mission est explicitement de favoriser la mobilité interrégionale, a constaté 
que, parmi les quelque 1000 jeunes qui s'établissent en région avec le soutien de son réseau, de plus en plus de migrants ne sont pas originaires du territoire de destination et, pour une proportion importante, sont originaires de grands centres urbains au sein desquels on retrouve une quantité croissante de Québécois issus de l'immigration. Selon les statistiques de l'organisation, cette perception renouvelée de ce que peut représenter le mode de vie en milieu rural rejoint de plus en plus de jeunes.

Il s'agit d'un résultat combiné du travail acharné de plusieurs intervenants, d'une certaine conjoncture économique et de l'apparition d'une nouvelle génération de jeunes aux valeurs semblables à celles des milieux ruraux (les générations $\mathrm{X}$ et $\mathrm{Y}$ accordent beaucoup d'importance au temps passé en famille, à la proximité de la nature, à la sécurité, au développement durable, etc.). Ainsi, plusieurs régions ont amélioré sensiblement leur solde migratoire.

Toutefois, rien n'est encore gagné! Une amélioration certes, mais trop peu pour assurer le renouvellement nécessaire des populations des milieux ruraux. Par ailleurs, l'écart avec les grands centres urbains est encore très présent, obligeant les régions à redoubler d'efforts pour compenser les mouvements migratoires dans un contexte, relativement nouveau celui-là, de rareté de main-d'œuvre qualifiée et de relève d'entreprise.

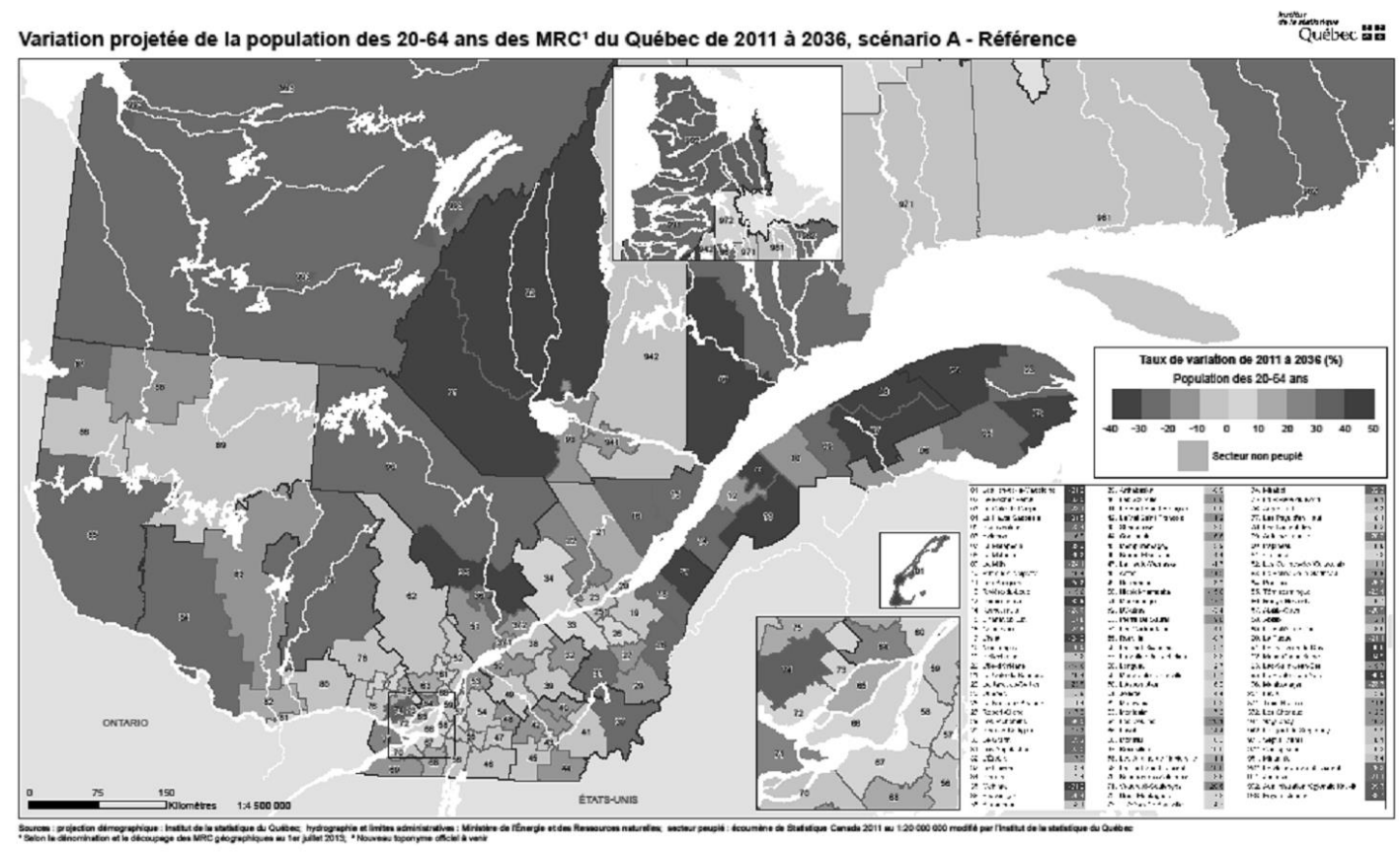

\section{En somme, l'avenir de l'ensemble du Québec passe par une contribution optimale de sa jeunesse, particulièrement des jeunes Québécois issus de l'immigration, à l'occupation et à la vitalisation du territoire.}

Dans cet esprit, chaque jeune Québécois, sans égard à son lieu d'origine, qui souhaite vivre et prospérer en région peut et doit contribuer au grand projet que représentent l'occupation et la vitalité des régions. Cependant, le processus migratoire ne peut plus être appréhendé de façon unidimensionnelle, mais plutôt dans une perspective de service à la clientèle ou, en d'autres mots, d'un accompagnement flexible tenant en compte les parcours de vie des différents types de clientèles (ex. : ruraux d'origine, urbains d'origine, immigrants) sur des territoires où cohabitent l'urbain et le rural.

En somme, l'avenir de l'ensemble du Québec passe par une contribution optimale de sa jeunesse, particulièrement des jeunes Québécois issus de l'immigration, à l'occupation et à la vitalisation du 
territoire. Nous croyons fermement que ces jeunes formés et dynamiques représentent un capital humain pour les communautés, et que celles-ci ont un potentiel d'accueil qu'il faut canaliser et optimiser. L'apport de ces jeunes Québécois dans une communauté, particulièrement dans les MRC à caractère rural, a des répercussions énormes sur une multitude de détails de la vie quotidienne, tels que la sécurité publique (présence de pompiers volontaires), le financement des services publics, la démocratie (pluralité des points de vue), le développement économique (création d'entreprises et expertise acquise à l'international), etc.

Ironiquement, force est de constater qu'aujourd'hui plus que jamais, une grande partie de ce capital humain réside dans les grands ensembles métropolitains. La mise en valeur et la promotion du vivre et prospérer en région demeurent essentielles, pour les régions bien entendu, mais aussi pour les jeunes urbains, particulièrement ceux issus de l'immigration, qui ne profitent pas d'un bassin suffisant d'occasions d'emploi. Dans les circonstances, il apparaît hautement pertinent de recentrer l'accompagnement à la mobilité interrégionale et même de soutenir son développement.

\section{LA CONTRIBUTION DES IMMIGRANTS AU DÉVELOPPEMENT DU QUÉBEC ET LEURS DÉFIS D'INTÉGRATION}

Le Canada et, parallèlement, le Québec sont deux des très rares États qui sélectionnent leurs immigrants. Dans un contexte mondial de concurrence pour l'attraction des talents, les immigrants qui se présentent à nos portes doivent être considérés comme des ressources de grande valeur. Les immigrants sélectionnés par le Québec pour leur profil de compétences et pour leur expertise ont donc des attentes, pour l'essentiel, très légitimes quant à leur intégration socioéconomique et à la concrétisation de leur désir de contribuer à leur société d'accueil. Ainsi, la migration devient de plus en plus l'incarnation d'un projet de vie et d'une stratégie d'ascension sociale basée sur le cumul d'un capital mobilitaire et, de moins en moins, sur une fatalité. Qualifiés, débrouillards et entreprenants, ils sont arrivés récemment ou sont de deuxième, voire de troisième génération et en quête d'un emploi qualifié.

Deux caractéristiques du profil de l'immigration au Québec attirent notre attention. La première
Cela dit, bien conscients du défi financier que doit relever le gouvernement du Québec, nous considérons néanmoins comme essentiel d'optimiser les structures et les ressources déjà en place, et d'assurer leur efficacité et leur cohésion.

Par ailleurs, compte tenu de l'évolution de la demande au cours des cinq dernières années, il est grandement nécessaire de mieux adapter le travail de liaison dans les grands centres ainsi que l'offre d'accompagnement à la mobilité aux différentes clientèles, telles que les jeunes Québécois issus de l'immigration et les entrepreneurs en devenir, et ce, dans une perspective de résultats tangibles pour les jeunes, les régions et le Québec en général.

De même, il apparaît de plus en plus nécessaire que l'État québécois ait avantage à se doter d'une vision stratégique de croissance démographique (androgène et exogène) à l'échelle nationale appuyée d'un plan d'action en mobilité interrégionale et, dans l'opérationnalisation, à s'associer à des partenaires et à des initiatives concrètes, axées sur de véritables résultats pour les migrants et pour l'ensemble des régions du Québec. est que $79,2 \%$ des personnes issues de l'immigration déclarent connaître le français, selon le ministère de l'Immigration, de la Diversité et de ''Inclusion (MIDI) $^{1}$. Chez les jeunes travailleurs qualifiés admis en 2013, $72,3 \%$ d'entre eux déclarent connaître le français. Cela constitue un critère essentiel lorsque le projet de migration d'une région métropolitaine vers une région à caractère rural québécoise est envisagé. L'autre caractéristique, donnée particulièrement intéressante pour les employeurs québécois qui sont en démarche d'internationalisation, est le taux de bilinguisme des immigrants au Québec : 57,5\%. Ce taux est de $15 \%$ supérieur à celui des jeunes Québécois d'origine. À notre avis, il s'agit d'une ressource à ne pas négliger, bien au contraire, car, à en croire les démographes, à partir de 2015, les immigrants représenteront la totalité de la croissance de la population active, devenant ainsi des acteurs majeurs pour l'ensemble du développement socioéconomique. 
Ainsi, une saine planification et la synchronisation des interventions sur les plans de la promotion, de la sélection et, surtout, de l'intégration des immigrants apporteront leur lot d'avantages pour tous, de la communauté d'accueil en passant par les employeurs, l'immigrant lui-même, sa famille jusqu'à son pays d'origine (351 milliards de dollars en transferts de fonds en 2001 par rapport à 107 milliards de dollars en aide publique au développement international à l'échelle mondiale) ${ }^{2}$.

Pourtant, les candidats issus de l'immigration, notamment les nouveaux arrivants sélectionnés par le Québec, ont certaines difficultés à trouver un emploi à Montréal. Malgré leur bonne volonté, les immigrants font face à un taux de chômage qui demeure gonflé à près du double de celui des Québécois d'origine, soit $11,5 \%$ contre $7 \% 3$.

Devant ce défi d'intégration socioéconomique, ceux-ci rivaliseront d'ingéniosité et adopteront différentes stratégies pour atteindre leur objectif et pour favoriser leur intégration à la société québécoise. La migration en région est, depuis 2008, une stratégie de plus en plus utilisée. D'autres stratégies, dont la déqualification temporaire dans le but spécifique d'acquérir l'expérience québécoise tant demandée par les employeurs ou une réorientation par un retour aux études, doivent, à notre avis, interpeler tous les acteurs en employabilité afin que ces stratégies d'intégration ne se traduisent pas en perte de capital de compétences. La recherche de stages, par exemple par le biais du programme Interconnexion, et l'optimisation des réseaux sociaux viendront compléter l'arsenal du jeune immigrant ${ }^{4}$.

Cela dit, alors que l'immigration pourrait améliorer considérablement le niveau d'occupation et de vitalité des régions à caractère rural, il en est tout autrement. En effet, près de $80 \%$ des quelque 50000 nouveaux arrivants annuels, dont près de $70 \%$ ont moins de 35 ans $^{5}$, s'établissent chaque année dans la grande région métropolitaine de Montréal, comparativement à un très mince $1,9 \%$ dans les 7 régions ressources du Québec. Ainsi, à peine 943 des 49489 nouveaux immigrants en 2009 projetaient de s'établir dans l'une ou l'autre des 7 régions ressources du Québec. En contrepartie, et à elles seules, les îles de Montréal et de Laval attiraient 38414 immigrants. Les statistiques récentes démontrent que les initiatives de régionalisation de l'immigration telles qu'elles sont appliquées peinent à renverser la métropolisation de l'immigration.

\begin{tabular}{|c|c|}
\hline $\begin{array}{c}\text { IMMIGRANTS ADMIS DE 2001 À 2010 } \\
\text { PRÉSENTS EN 2012 }\end{array}$ & $\begin{array}{c}\text { IMMIGRANTS ADMIS DE 2003 À 2012 } \\
\text { PRÉSENTS EN 2014 }\end{array}$ \\
\hline Taux de présence 75,9\% & Taux de présence 75,7\% \\
\hline Résidents de la RMM6 75,2\% & Résidents de la RMM 74,9\% \\
\hline Résidents de la CMM7 80,7\% & Résidents de la CMM 80,8\% \\
\hline
\end{tabular}

Source : Résultats de l'enquête auprès des immigrants de la catégorie des travailleurs qualifiés (ETQ) - Gouvernement du Québec, 2013

L'intégration des immigrants habitant dans les petites régions moins urbanisées est meilleure que dans les grands centres, et cet avantage s'accentue au fil des ans.

Selon une étude de 2008 de Statistique Canada, il en ressort que l'intégration des immigrants habitant dans les petites régions moins urbanisées est meilleure que dans les grands centres, et cet avantage s'accentue au fil des ans. L'étude définit l'intégration économique selon deux aspects: l'écart initial de revenu au moment de l'établissement entre les immigrants et les Canadiens, puis la vitesse de convergence ou le rattrapage subséquent à mesure que les années passent.

Dans les très grandes régions urbaines, précise cette étude, l'écart initial de revenu est de $37 \%$. Après quatre ans, il est de $22 \%$, et il faut attendre 
la douzième année pour qu'il tombe sous le seuil de $10 \%$. En revanche, dans les plus petites villes situées en région, l'écart initial n'est que de $14 \%$ et, dès la quatrième année, les immigrants gagnent $2 \%$ de plus que les Canadiens. L'avantage relatif des immigrants continue d'augmenter au cours des années pour atteindre un sommet de $18 \%$ à la onzième année 6 . Ainsi, bien que les immigrants aient au départ un revenu plus faible dans tous les types de régions, l'écart se rétrécit à mesure qu'on se déplace le long du gradient d'urbain à rural.

\section{L’intégration des immigrants est plus rapide dans les plus petites régions}

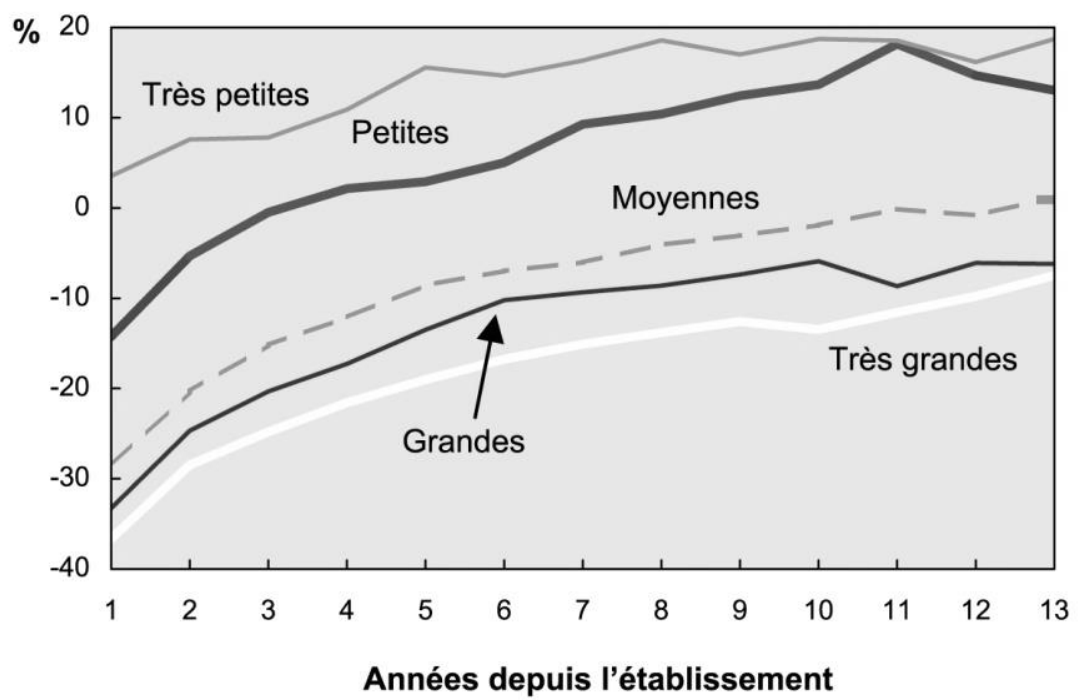

Source : Statistique Canada, banque de Donnees administratives longitudinales, 1992 a 2005

En y regardant de plus près, on constate qu'il devient évident qu'une meilleure intégration des immigrants au Québec, notamment par l'amélioration du processus de régionalisation, servirait plusieurs causes. Celle de l'immigrant d'abord, celle de la communauté et, certainement, celle de l'économie du Québec, car une meilleure intégration des immigrants amènerait une augmentation du PIB de 2,1\%. S'il est devenu incontournable de rétablir la santé financière du Québec pour assurer une certaine équité intergénérationnelle, il y a certes la rationalisation des dépenses, mais aussi - et surtout - la rentabilisation des actifs. Comme le mentionnait le ministre délégué aux Petites et Moyennes Entreprises, à l'Allègement réglementaire et au Développement économique régional, monsieur Jean-Denis Girard, il n'y a pas que la colonne des dépenses à scruter : il y a aussi la colonne des revenus à gonfler.

Dans cet esprit, nous sommes d'avis que :
« la responsabilité du pouvoir gouvernemental ne se limite pas à l'octroi de deniers publics, mais implique aussi une réflexion fondamentale sur l'évolution de la société québécoise et son fonctionnement actuel. Encore plus important, le gouvernement détient un rôle d'accompagnement de la population québécoise vers l'atteinte des objectifs de société. Dans le cadre de la régionalisation de l'immigration, il est indispensable que l'implication du gouvernement provincial devienne plus stratégique afin que ce projet puisse transcender le discours politique et devienne une réalité sociale 7 . »

Car, bien que l'immigration ne soit pas à elle seule une panacée pour résoudre les maux du Québec, sa contribution au développement économique et social serait plus substantielle si les actions de régionalisation, les programmes d'adaptabilité socioprofessionnelle et les mesures de lutte contre la discrimination étaient bonifiés. 


\section{L'INTÉRÊT CROISSANT DES IMMIGRANTS POUR L'ÉTABLISSEMENT EN RÉGION}

C'est donc dans ce contexte que les jeunes immigrants montréalais se tournent du côté des régions. Bien que la recherche d'un emploi stimulant demeure le principal facteur de mobilité interrégionale, il apparaît que les immigrants recherchent dans les milieux de vie périurbains et ruraux les mêmes caractéristiques que les natifs (un emploi de qualité, la proximité des réseaux sociaux, la tranquillité, l'accès à la nature et aux loisirs, le faible coût de la vie, etc.).

Dans les faits, depuis 2006-2007, la clientèle de Place aux jeunes en région issue de l'immigration a quadruplé. Les statistiques du point de service de PAJR à Montréal témoignent déjà très clairement de cette réalité, et il est devenu évident que c'est ce segment de sa clientèle qui connaîtra la croissance la plus soutenue au cours des prochaines années.

Évidemment, le resserrement du marché de l'emploi dans la métropole à la suite de la crise financière de 2008-2009 a eu un effet considérable sur la migration des jeunes Québécois issus de l'immigration, pour qui les possibilités d'emploi en région leur semblent plus accessibles.

Clientèle immigrante chez PAJR

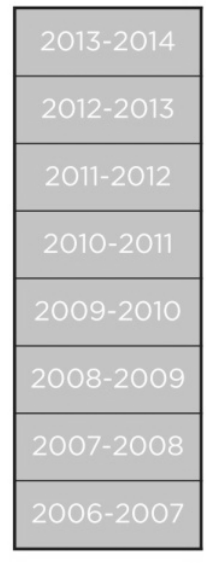

SÉJOURS

1096

1247

1210

\begin{tabular}{|l|l|l|}
\hline 1173 & 434 & $37 \%$ \\
\hline 1212 & 372 & $31 \%$ \\
\hline 885 & 199 & $22 \%$ \\
\hline 875 & 115 & $13 \%$ \\
\hline 961 & 114 & $12 \%$ \\
\hline
\end{tabular}

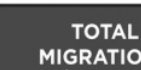

MIGRATIONS \begin{tabular}{l|l} 
IMMIGRANTS & $\begin{array}{l}\text { POURCENTAGE } \\
\text { IMMIGRANTS }\end{array}$
\end{tabular} IMMIGRANTS

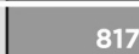

466

$43 \%$

480

\begin{tabular}{|l|l|}
\hline $39 \%$ & \\
$37 \%$ &
\end{tabular}

\begin{tabular}{l|l|l}
451 & $37 \%$ & 985 \\
\hline
\end{tabular}

896

777

945

965

1130

\section{$s^{2}$}




\section{Caractéristique importante cependant, le projet de migration des néo-Québécois n'implique pas uniquement leur besoin de trouver un emploi.}

Caractéristique importante cependant, le projet de migration des néo-Québécois n'implique pas uniquement leur besoin de trouver un emploi. Dans leur cas, il implique aussi - et surtout - le choix de la bonne communauté d'accueil, une certaine préparation aux particularités de la vie en région, de même que la nécessité de faire des démarches administratives parfois lourdes pour les candidats. Ainsi, pour PAJR, la clientèle issue de l'immigration n'est pas seulement plus nombreuse; elle est également différente dans ses besoins et sa complexité.

Cependant, des candidats immigrants se disent moins satisfaits que les non-immigrants des services d'intégration offerts. Plusieurs facteurs peuvent expliquer ces insatisfactions. Nous en nommons ici quelques-uns. D'abord, la notion espace-temps imaginée par le nouvel arrivant est peu concordante avec la réalité et la quantité d'information que celui-ci doit assimiler. De plus, il doit démêler un nombre de structures et de mandats associés, et les exigences des ordres et des corporations de métiers, qui sont nouvelles pour lui. Il y a aussi l'exigence de l'expérience québécoise, qu'il doit déchiffrer afin de convaincre les employeurs qu'il a les compétences souhaitées, et la nécessité parfois de retourner sur

\section{UN PARCOURS DE COMBATTANT}

Depuis quelques années, les structures locales ou régionales et les projets se sont multipliés pour tenter de répondre à une demande sans cesse croissante des milieux pour des actions favorisant l'occupation dynamique des territoires. Cependant, force est de constater que l'expertise en mobilité interrégionale et l'offre de services en médiation interculturelle ne se sont pas développées de façon optimale.

Aujourd'hui, la volonté du gouvernement du Québec de régionaliser l'immigration se traduit sur le terrain par une multiplication d'interventions et les bancs d'école pour acquérir les normes et les outils du marché du travail québécois.

On trouve aussi la méconnaissance du territoire québécois et les préjugés véhiculés sur celui-ci; les réticences des employeurs qui n'ont pas acquis la compétence interculturelle; et, surtout, le stress occasionné par l'urgence de trouver un emploi, car les économies fondent comme neige au soleil! Ainsi, les candidats issus de l'immigration ont des attentes plus élevées. Malgré tout, persévérants, ils investiront temps et énergie pour relever le défi et pour contourner les obstacles rencontrés.

Et c'est sans parler du défi de l'intégration et de l'enracinement dans la communauté d'accueil. Car, si toutes les communautés ne sont pas au même niveau d'acceptation de la diversité culturelle, toutes, sans exception, souhaitent un avenir prometteur pour leur population. Il y a donc là quelque chose sur quoi bâtir! Les élus et les acteurs socioéconomiques sont donc aux premières loges et doivent accompagner leur collectivité pour l'accueil des immigrants et pour la reconnaissance de l'apport de la diversité culturelle.

D'autant plus qu'à l'heure où l'entrepreneuriat apparaît comme l'une des options de création de richesses et d'optimisation du capital humain dans l'ensemble de la province, il est important de noter que 19 à $23 \%$ des Québécois issus de l'immigration sont des travailleurs autonomes ou entrepreneurs individuels, comparativement à $11,5 \%$ pour l'ensemble des Québécois.

de structures spécifiquement mises en place pour assurer l'accompagnement des immigrants dans leurs démarches d'intégration, notamment à travers le prisme de la régionalisation. À titre d'exemple, en plus de PAJR qui œuvre auprès de l'ensemble des jeunes Québécois sur l'essentiel du territoire, il existe, au sein de la Table de concertation des organismes au service des personnes réfugiées et immigrantes (TCRI), une soixantaine d'organismes qui sont financés par le gouvernement pour intervenir exclusivement auprès des immigrants, dont au moins quatre ont un mandat ou une partie de mandat lié spécifiquement à la régionalisation. 
S'ajoutent à cela une vingtaine d'organisations situées en région et autres organisations régionales qui interagissent aussi avec les immigrants, soit à la Grande Bibliothèque de Montréal, soit dans des organisations partenaires.

Dans les faits, à partir du moment où le jeune Québécois issu de l'immigration qui réside à Montréal envisage de migrer en région, il sera, dans la plupart des cas, dirigé vers l'atelier Objectif Intégration du MIDI, programme offert par l'un des quatre organismes de Montréal. De là, ceux-ci vont rediriger le jeune immigrant parfois vers l'agent de liaison de PAJR de Montréal ou de Québec, parfois directement vers l'agent de migration en région, parfois vers un organisme local d'accueil et d'insertion des immigrants, parfois vers un organisme d'employabilité en région.

Dans bien des cas, cependant, alors que PAJR ne fait aucune promotion spécifique auprès de cette clientèle, le jeune immigrant s'adressera directement à PAJR, dans le cadre de sa stratégie d'intégration économique, afin d'être accompagné vers une région du Québec.

\section{Les jeunes immigrants représentent près de $40 \%$ des participants aux séjours exploratoires, service emblématique de découverte d'une MRC offert depuis 25 ans, mais constituent seulement $15 \%$ des migrations réussies.}

Cela dit, dans une proportion importante des cas, nous observons que les candidats qui s'adressent directement à PAJR ne sont pas prêts à entamer un parcours migratoire (niveau de francisation faible, profil professionnel non adéquat, situation personnelle ou familiale ambiguë et, surtout, méconnaissance des réalités régionales/rurales, etc.). Cela rend le projet de l'immigrant très hasardeux et diminue de beaucoup les chances de résultats positifs, pour lui-même et pour la communauté d'accueil. Dans les faits, chez PAJR, les jeunes immigrants représentent près de $40 \%$ des participants aux séjours exploratoires, service emblématique de découverte d'une MRC offert depuis 25 ans, mais constituent seulement $15 \%$ des migrations réussies.

En somme, le manque de cohésion des différentes ressources en place induit de la confusion pour l'usager qui, dans sa volonté réelle de se trouver un emploi et un milieu de vie stimulant pour contribuer au développement du Québec, ne semble pas savoir ni à qui, ni quand, ni comment s'adresser pour obtenir de l'aide dans son projet de migration. Par ailleurs, malgré toute la bonne volonté des professionnels impliqués, cette confusion favorise un dédoublement inefficace des interventions auprès du migrant.

Alors qu'elles devraient travailler en collaboration au bénéfice de l'immigrant et des collectivités régionales, dans une perspective de complémentarité ou d'intégration, PAJR et les ressources financées par le MIDI en arrivent malheureusement à se concurrencer par leur offre de services.

Notre analyse nous a permis d'identifier 9 services similaires dans la chaîne logique de prestations, dont la transmission d'informations sur les différentes régions et sur la vie en région; l'accompagnement individualisé selon le profil et la région choisie; la mise en contact avec le réseau de correspondants en région; les offres d'emploi des employeurs des régions; la révision des techniques de recherche d'emploi adaptées aux régions. Et la liste continue!

Même constat du côté des activités de financement locales et régionales, qui sollicitent bien évidemment les mêmes partenaires, diminuant d'autant pour chacune des structures l'appui financier de proximité et l'ancrage territorial.

En résumé, il apparaît que, collectivement, l'usage des ressources, des énergies et des compétences n'est pas optimal. Par conséquent, il en découle un résultat somme toute très décevant sur le plan de la régionalisation de l'immigration dans les diverses régions du Québec. 


\section{RECENTRER LES SERVICES DE MOBILITÉ INTERRÉGIONALE}

Si les conséquences annoncées du vieillissement accéléré des populations rurales ne justifiaient pas à elles seules une réévaluation sérieuse des services de régionalisation de l'immigration, la situation des finances publiques, elle, nous obligerait à le faire. Dans les circonstances, il ne s'agit certainement pas de remettre le chantier en question, mais plutôt de réfléchir à une optimisation des ressources à travers une démarche recentrée de mobilité interrégionale ciblant l'ensemble des jeunes Québécois : ruraux d'origine, urbains et immigrants.

Bien entendu, il s'avèrera extrêmement important d'assurer, dans cette démarche, une sensibilité à la réalité vécue des jeunes immigrants de même qu'une approche respectueuse des besoins et des attentes des territoires d'accueil et employeurs potentiels.

Cependant, avant même de se questionner sur le processus de régionalisation de l'immigration, soulignons que, pour plusieurs experts et praticiens $\mathrm{du}$ domaine, les bas taux de réussite de la régionalisation s'expliquent, en partie du moins, par un manque en amont du processus d'immigration. Nous proposons ici de réfléchir à une mesure simple qui pourrait être rapidement mise de l'avant. L'outil SIEL (outil Objectif Intégration du MIDI en ligne), qui a déjà un court module sur les régions, pourrait contenir une section Mobilité interrégionale où, avant même d'immigrer, l'immigrant pourrait, s'il a les atouts de base déjà mentionnés, planifier une migration en région afin que par la suite, dès son arrivée au Québec, il se mette en mouvement vers la région, la MRC ou la municipalité ciblée.

Arrivé au Québec, l'immigrant doit pouvoir rencontrer une personne ayant le mandat de l'accueillir et de l'amener à réaliser un bilan personnel, première étape en vue d'établir une stratégie d'intégration adéquate et personnalisée. Le

\section{CONCLUSION}

De notre point de vue et si nous considérons les ressources financières limitées de l'État, le Québec dispose d'ores et déjà de l'essentiel des composantes nécessaires à la mise en œuvre d'une véritable stratégie de déploiement de l'immigration en région. statut légal régularisé, la connaissance du français et la reconnaissance des diplômes ou plutôt de l'expertise deviennent des incontournables. Les étapes subséquentes touchent l'employabilité au sens large et la connaissance générale du marché du travail québécois. La séance de l'atelier Objectif Intégration, d'une durée de 24 heures, qui présente aux nouveaux arrivants la dynamique et la culture du marché de l'emploi au Québec, les compétences exigées par les employeurs et les stratégies et outils efficaces pour la recherche et l'obtention d'un emploi, demeure un excellent outil.

Sans être exhaustif, ce type d'intervention extrêmement pertinent permet d'outiller l'immigrant à intégrer la société québécoise de façon générale. Un tel bilan personnel bonifié ouvre la porte à une migration en région qui se présente, au-delà de la question de l'emploi, avec ses propres règles, pièges et défis, liés par exemple au choix de la bonne région (compatibilité sur les plans de la culture, de la morphologie, du transport, etc.).

Par la suite, il faut qu'il y ait prise en charge du reste $\mathrm{du}$ processus par l'accompagnement des immigrants qui souhaitent s'établir en région en créant un pont efficace avec les organismes communautaires d'accueil de la métropole. L'objectif devient alors de déterminer et de mettre en œuvre un plan individuel de migration durable impliquant une stratégie d'intégration dans la communauté d'accueil.

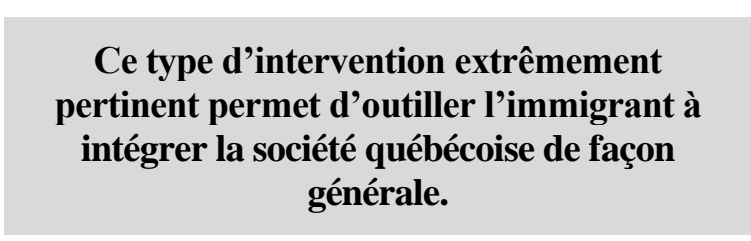

D'un côté, la valeur ajoutée de PAJR réside principalement dans sa structure d'accompagnement (plateforme électronique, outils d'accompagnement, produits et services à l'usager, réseau d'agents, structure intégrée, etc.) et dans son intelligence territoriale, c'est-à-dire 
sa connaissance fine des réalités terrain (emplois cachés, mouvement de la main-d'œuvre, projet de développement, etc.), notamment par le déploiement de son vaste réseau d'agents et de partenaires à l'échelle locale.

\section{Le défi est de changer de perspective pour considérer les immigrants non pas comme des cas particuliers, des cas lourds qu'il faut déplacer et nécessitant une structure spécifique, mais plutôt comme des Québécois engagés dans leur progression socioprofessionnelle.}

À l'inverse, PAJR n'a pas actuellement l'expertise à l'interne pour assumer un accompagnement adapté sur l'ensemble du parcours migratoire des Québécois issus de l'immigration. C'est pourquoi l'intelligence sectorielle en médiation interculturelle développée par certains praticiens est hautement pertinente et nécessaire afin d'actualiser l'offre de services en mobilité interrégionale. Plus concrètement encore, cette expertise se veut hautement nécessaire, en amont du parcours migratoire, en matière de préparation de base et, en aval du parcours, en matière d'accueil et d'encadrement adaptés, favorisant ainsi une migration et une intégration durables et profitables.

La tendance et l'intérêt marqué des candidats d'origine immigrante pour les régions s'étant confirmés, il est devenu incontournable que le Québec revoie et améliore son offre de services aux immigrants. C'est pourquoi un recadrage de l'action collective en faveur d'une meilleure prestation de services à l'usager et d'un plus grand impact en développement socioéconomique local est maintenant inévitable.

Le défi est de changer de perspective pour considérer les immigrants non pas comme des cas particuliers, des cas lourds qu'il faut déplacer et nécessitant une structure spécifique, mais plutôt comme des Québécois engagés dans leur progression socioprofessionnelle ayant, comme les ruraux d'origine et les urbains, des perceptions différentes des régions à caractère rural et des profils plus ou moins en phase avec le mode de vie qui y prévaut.

Le cas échéant, les objectifs seront de recadrer les orientations et le travail des ressources humaines déjà impliquées dans le secteur de la mobilité interrégionale (toutes catégories d'usagers confondues); d'actualiser l'offre de services à l'usager et aux communautés en intégrant les grands principes de l'approche client; et, enfin, de « recanaliser» les ressources financières actuellement éparpillées afin de créer un seul continuum intégré de services en mobilité interrégionale.

\section{BIBLIOGRAPHIE}

${ }^{1}$ MIDI (2014). Fiche synthèse sur l'immigration et la diversité ethnoculturelle au Québec, Direction de la recherche et de l'analyse prospective, gouvernement du Québec, 4 p., www.midi.gouv.qc.ca/publications/fr/ recherches-statistiques/FICHE_syn_an2013.pdf; MIDI (2104), Présence en 2014 des immigrants admis au Québec de 2003 à 2012, Direction de la recherche et de l'analyse prospective, gouvernement du Québec, 36 p., www.midi.gouv.qc.ca/publications/fr/recherchesstatistiques/PUB_Presence2014_admisQc.pdf.

${ }^{2}$ Wihtol De Wenden, Catherine (2013). La question migratoire au XXI ${ }^{\mathrm{e}}$ siècle : migrants, réfugiés et relations internationales, Paris, Presse de Sciences Po, 266 p.

${ }^{3}$ Grenier, André (2013). Le marché du travail au Québec : Évolution récente et perspectives, Conférence prononcée au Congrès national sur la migration des jeunes en région, Montmagny, Québec.

${ }^{4}$ Comité consultatif Jeunes (2013). L'inser-tion en emploi des jeunes immigrants de première génération, Montréal, 19 p., http://ccjeunes.org/IMG/ pdf/ccj_avis_ jeunes_immigrants_2013.pdf.

${ }^{5}$ MIDI (2014). Fiche synthèse sur l'immigration et la diversité ethnoculturelle au Québec, Direction de la recherche et de l'analyse prospective, gouvernement du Québec, 4 p., www.midi.gouv.qc.ca/ publications/fr/ recherches-statistiques/FICHE_syn_an2013.pdf.

${ }^{6}$ Bernard, André (2008). "Les immigrants dans les régions ", Perspective, Statistique Canada, p. 8, www.statcan.gc.ca/pub/75-001-x/2008101/pdf/10505fra.pdf.

${ }^{7}$ Poirier-Grenier, Geneviève (2007). Les politiques de régionalisation de l'immigration au Québec et leurs effets sur le système urbain, Mémoire, Université du Québec à Montréal. 